\title{
Camilo José Cela y la génesis de La Colmena
}

\author{
Camilo José Cela e a gêneses de A Colmeia
}

Camilo José Cela and the La Colmena's genesis

Adolfo Sotelo Vázquez

Facultad de Filología - Universidad de Barcelona - Barcelona - Espanha $\diamond$

Resumen: El presente trabajo describe brevemente el arte novelístico de Camilo José Cela entre la mirada y la memoria. A partir de dicha naturaleza de la novela, se examina la génesis externa de La colmena. Es decir, algunos de los estadios que el manuscrito atravesó a tenor de las diversas lecturas públicas que el autor hizo del texto en Madrid y en Barcelona durante los años 1945 y 1946.

Palabras clave: Memoria; Mirada; Censura

Resumo: O presente trabalho descreve brevemente a arte romanesca de Camilo José Cela entre la mirada e a memória. A partir da natureza do romance, se examina la génesis externa de $L a$ colmena. Ou seja, alguns dos circuitos que o manuscrito atravessou a propósito das diversas leituras públicas do texto que o autor realizou em Madri e em Barcelona, durante os anos 1945 e 1946.

Palavras-chave: Memória; Mirada; Censura

\begin{abstract}
This paper briefly describes the novelistic art Camilo José Cela between his glance and memory. From the novel's nature, examining the external genesis of La Colmena. Namely some of the circuits that the manuscript crossed the subject of several public readings of the text that the author held in Madrid and Barcelona during the years 1945 and 1946.
\end{abstract}

Keywords: Memory; Glance; Censorship

"Ni la literatura ni el hombre se hacen de mármol solemne"

(Camilo José Cela, 1976)

\section{I}

En varias ocasiones Camilo José Cela rechazó la ordenación o clasificación de los géneros literarios como una realidad consolidada de la que se puede inferir valoraciones y otras consecuencias. Desde su óptica de cronista, que no de crítico literario -cuyo trabajo sobre la obra de creación no le fascinó nunca- escribía en 1949:

El cronista insiste en su vieja idea de que admitir una apriorística ordenación de los géneros $\mathrm{y}$, lo que es más grave, sacar de ella rígidas consecuencias a ultranza, sería algo tan disparatado como medir a las mujeres por el tamaño o el nombre de pila (CJC, 1978, p. 224).
En proximidad con algunas de las reflexiones teóricas más inteligentes del siglo XX, Cela descree de los recetarios establecidos en torno a estéticas inútiles o convencionales, que postulan el equilibrio y desdeñan la verdad entrañable, íntima, la que contiene "la fluencia vital" que decía Ortega, la que el novelista ha digerido con sus cuatro estómagos:

La novela -escribe Cela en 1943- precisa de una verdad entrañable, de una verdad de cuerpo entero, de una verdad muy digerida por su autor. El novelista debiera tener cuatro estómagos, como los bueyes: panza, bonete, libro y cuajar. Con un sistema así estaría siempre rumiando esa verdad y la novela saldría mejor, más acabada. Con cuatro estómagos no hay quien se atreva a hacer equilibrios (CJC, 1978, p. 85). 
En este sentido, Cela parece acercarse a una reflexión que Unamuno expone colateralmente en el importante "Prólogo" a Tres novelas ejemplares y un prólogo (1920), donde sostiene que Balzac - paradigma del gran novelistano sólo tomaba notas de lo que veía y oía, sino que "llevaba el mundo dentro de sí" (UNAMUNO, 1990, p.56). Con el mundo dentro de sí, con la idea de novela atesorada en el alma y en el cuerpo, Cela ha abordado un género literario proteico, cuyo denominador común es el contar y en el que se permiten todo tipo de extravagancias y de libertades, según Baroja dejó dicho en diversos lugares.

La ventana del escritor, su corazón, se abre sobre cualquier paisaje (tan solo en el dominio de la novela y desde Pascual Duarte a La colmena, son bien distintos) $\mathrm{y}$ vuelca su memoria sobre un ancho panorama que tiene diversos caminos que, a veces, "están erizados de zarzas que nos hieren y nos desgarran las carnes" (CJC, 1978, p.36). Mirando desde la ventana, mirando desde el corazón, el escritor se confiesa, purga su adentro. Y así Pascual es la acción desde la confesión, Pabellón de reposo es la inacción desde la confesión, Nuevas andanzas $y$ desventuras de Lazarillo de Tormes es el palimpsesto desde la confesión, La colmena es la mediocridad la vulgaridad, lo gris y lo tibio, de una sociedad y de una ciudad desde la crónica untada de confesión, y Mrs Caldwell es un doloroso esfuerzo poético desde la confesión. Son diversas digestiones del novelista y son también facetas diferentes de la misma verdad íntima.

La memoria es una potencia del alma que anuda toda la obra de Cela. Y la memoria se materializa en dos dimensiones: la duración y la novela. En el primer caso, Cela ha aceptado plenamente la reflexión de Bergson según la cual, el yo no es más que la condensación de la historia que hemos vivido, el presente del yo está conformado con la continua co-presencia del pasado.

Cela, como otros grandes novelistas del siglo XX (Marcel Proust y William Faulkner), ha conformado su obra narrativa desde la convicción -a la que se refieren sus prólogos a los dos tomos de memorias, $L a$ rosa y Memorias, entendimientos y voluntades, y algún apunte aislado como "La herramienta de la memoria" $(17-\mathrm{V}-1992)^{1}$ - de que con la memoria se convive o se malvive porque es una de las estructuras fundacionales del yo, y así -tal reza un cuento de 1950 recogido en Baraja de invenciones (1953)- la memoria es la fuente del dolor y la experiencia del vivir su poso.

La duración, el continuar siendo, se nutre de la memoria y deviene en temporalidad y en novela (arte del tiempo), a la vez que en discurso factual que encuentra

\footnotetext{
1 Cf. "Dan rabia, pero también dan risa, las piruetas de la memoria sobre el borroso o tenue telón de fondo del tiempo; estas volteretas serían, a no dudarlo, un magnífico ingrediente para cocinar literatura" (CJC, A bote pronto, p. 43).
}

el cauce de los libros de memorias. Desde La familia de Pascual Duarte en el arte narrativo de Cela se advierte el alimento continuado del tiempo y de las campanadas que lo miden y lo amasan en la memoria. Mediante la memoria se puede iluminar un determinado momento histórico con mayor intensidad que a través de la reconstrucción histórica, dibujando la morada vital de una colectividad, de un medio y de un momento: Pascual Duarte, La colmena (aun a pesar de su radical modelo de "crónica", no deja de advertirse en ella el pulso de la memoria), San Camilo, 1936, Mazurca para dos muertos o Madera de boj. Intensamente bronca y amarga la confesión de Pascual, la memoria de Pascual y sus añadidos textuales (constitutivos de la novela) revelan la intrahistoria latente de una colectividad que, abocada a la guerra civil, la hizo morada vital durante tres años. Este nieto del 98 se acercó tanto en su primera novela como en la genial creación del apunte carpetovetónico al descubrimiento de los factores que delimitaban la vividura y configuraban la morada vital del hombre ibérico. Ningún otro sentido tiene su rigurosa afirmación del ensayo "Fauna carpetovetónica" (1949):

\footnotetext{
Aun a riesgo de caer en el tipismo -que tampoco, bien mirado, habría de considerarse una desgraciamerecería la pena pararse un punto a clasificar nuestra fauna humana: esa esquina en la que un Cardenal Cisneros puede nacer al lado de un Pascual Duarte, un Hernán Cortés a la vera de un Lagartijo, una reina Isabel a orillas de una Chelito, y un Cid Campeador codo con codo con un Tomás de Antequera y perdón por la manera de señalar.

Si la variedad es la riqueza -como parece bastante probable- nuestra fauna humana, nuestra hirsuta y violenta fauna carpetovetónica, podría hacernos riquísimos de sugerencias (CJC, 1979, p. 36).
}

De esa fauna hispánica y de la experiencia, de la vivencia, de la guerra civil nace el Pascual. La memoria inmediata, desaforada y amarga, le impulsó a crear a un habitante representativo de una morada vital, cuya agónica y trágica configuración se acababa de vivir. Desde el insuficiente reino de las ideas Cela también acabó por saberlo:
Esta característica de la guerra civil latiendo en cada pecho, es una de las determinantes más concretas del español y uno de los prismas a cuya luz puede verse, con mayor claridad, aquello que llamamos lo español. La discordia civil, esa cruenta e impolítica maldición que pesa sobre España, anida como un fiero aguilucho en los más recónditos entresijos de cada español que, cuando no está contento consigo mismo, se pelea consigo mismo en el espejo de los demás. (CJC, 1978, p. 619)

Aunque Cela haya escrito que "la memoria es más fiel, más concreta, más dibujadora que nuestros propios 
ojos" (CJC, 1978, p. 19-20), lo cierto es que junto al papel fundamental de la memoria está el de la mirada. De ella nace la novela como "crónica" ("no perdamos la perspectiva" reza el principio de La colmena) y el libro de viajes, que el Viaje a la Alcarria, bajo un aparente behaviorismo deja traspasa lo emocional y lo subjetivo. La mirada que articula la construcción intrahistórica de La colmena no es tampoco objetiva, está decantada por el fondo sentimental del novelista. Se trata, en ambos casos, de una mirada en la que radica la paradoja señalada por tantos críticos a propósito de la novela (Robert Spires o Gonzalo Sobejano) ${ }^{2}$ y acerca del Viaje a la Alcarria por Pozuelo Yvancos, quien ha escrito: "en esa contradicción aparente, en esa dialéctica entre lo real cotidiano narrado con frugal economía objetivizadora y lo emocional subjetivo, veo una razón más de su singularidad"3. Dicha mirada tiene unas características que ha explicado el propio Cela en un artículo barcelonés de 1952: "Elogio del mirón”. El mirón tiene una doble faceta: mira estrenando unos ojos cada mañana y necesita de esa mirada para escuchar su propio adentro, su propia mismidad (de la que forma parte la memoria):

El mirón -que no es el observador, ni el espectador y ni siquiera el contemplador- es el hombre con alma de árbol que necesita ver la vida de los otros hombres para poder escuchar, casi como sin querer, el tímido latido de su propio corazón. El mirón es el hombre espejo -por eso se asusta cuando se ve reflejado en la luna de los escaparates-, el hombre que vive en los demás -en éste, en aquél o en aquel otro de más allá-, el hombre que en los momentos de tránsito llega a olvidarse de sus mismas carnes para ser, según la exacta expresión del pueblo, todo ojos (CJC, 1978, p. 224-225).

Ese hombre que es "todo ojos" se pasma ante lo que la realidad le muestra; su mirada desnuda la realidad y al mismo tiempo le identifica. Por ello el arte del mironismo descubre tanto la intrahistoria del mundo en torno como el fondo sentimental del propio mirón. Ese viajero que pasa de madrugada junto a las tapias del Retiro, iniciando su vagabundaje, desea un mirar desnudo, quiere un ver de mirón: "piensa rascar el corazón del hombre del camino, mirar al alma de los caminantes asomándose a su mirada como al brocal de un pozo". (CJC, 1965, p. 37)

El arte del mirón resulta así un arte objetivo con honda participación del fondo sentimental del que mira, descubierto precisamente en cada una de las miradas que coloca ante sus ojos lo aparentemente anodino de la vida: "una señora a la que le dio un desmayo, una niña rubita que salta a la comba, un gorrión que come despreocupadamente unas migas de pan" (CJC, 1978, p.225), ateniéndonos a los ejemplos que el propio Cela propone en su "Elogio del mirón".
Cela aceptó en la fragua de su novela la mirada y la memoria, la confesión, la letanía y la crónica como caminos imprescindibles para conformar unos objetos estéticos fuertemente apegados a la morada vital de los españoles. Su arte literario y, en concreto, su novela, es en cierto modo, su vividura, sin que ello suponga despegarse un ápice del deber complementario del novelista contemporáneo, que según uno de sus artículos de 1952 es "actualizar los temas que no han envejecido y vivificar la savia de los mitos eternos" (CJC, 1978, p. 16).

\section{II}

Cela empezó a escribir La colmena en Madrid en $1945^{4}$, al tiempo que publica tres volúmenes: de poesía, Pisando la dudosa luz del día. Poemas de una adolescencia cruel (Zodiaco, Barcelona); de narraciones, Esas nubes que pasan... (Afrodisio Aguado, Madrid); y una recopilación de sus primeros artículos, Mesa revuelta (Sagitario, Madrid). El joven maestro trabaja con constancia y tenacidad, y en una versión incompleta presenta la novela que ya se llama La colmena (a lo largo de la redacción inicial del año 45 se tituló "Café europeo", primero, y "Café La Delicia", después, y más tarde, "La ciudad llagada") a la censura el 7 de enero de 1946. La censura la prohibió y el propio escritor instó al editor, Carlos F. Maristany de Ediciones del Zodíaco de Barcelona, que le acababa de publicar el poemario Pisando la dudosa luz del día y que le publicaría durante el 46 la cuarta edición de La familia de Pascual Duarte, a presentar el texto La colmena, primera parte del libro primero, Caminos Inciertos, de La Tarea infinita (Novela), como libro de bibliófilo para poder salvar los escollos de la censura. En 1965 CJC recordaba lo sucedido: "El 27 de febrero solicitó el editor el oportuno permiso para una tirada con características especiales, de lujo y reducida; fue también denegada, en oficio del 9 de marzo" (CJC, 1978, p. 140).

Como ha mostrado Fernando Huarte Morton ${ }^{5}$ (2000, p. 105-154) el texto presentado a la censura terminaba

2 Cf. Robert C. Spires, "Cela's La colmena: The Creative Process as Message", Hispania, 55 (1972), pp. 873-888. Y Gonzalo Sobejano, "Prólogo" a CJC, La colmena, p. 15-16.

3 José María Pozuelo Yvancos, "Introducción" a CJC Viaje a la Alcarria, p. 35. Cf. también Adolfo Sotelo Vázquez, "El retorno obligado. Viaje a la Alcarria de Camilo José Cela", El Observador (3-VIII-1991).

$4 \mathrm{Si}$ no ando errado la primera referencia que CJC hace a su futura obra maestra es la que procede del epistolario con Carlos F. Maristany (3-I1945): "Tengo notas tomadas para una novela a la que quizás titule $\mathrm{La}$ clientela de María Domingo".

5 De este trabajo aprovecho su abundante y fidedigna información. A ella se debe añadir la procedente del epistolario de Cela. Una carta del escritor a Carlos F. Maristany (2-I-1946) nos informa: "El primer volumen -mejor sería decir, la primera novela de la serie- la tengo ya terminada y a tu disposición. La titulo La colmena y quizás el lunes la presente ya en Censura." El borrador de una carta -que no se llegó a cursar-dirigida por CJC al editor Saturnino Calleja de Ediciones "La Nave" (14-I-1946) complementa la noticia: "He acabado una novela que titulo La colmena que, dadas sus características, no encaja en 'La Nave'." 
con la fecha "Madrid, diciembre, 1945" y se trata en efecto de una versión incompleta, de la que interesa destacar que la historia se data en 1943, momento acorde con la acción de la novela, pese a que con posterioridad CJC ha sostenido siempre que dicha historia discurría en el Madrid de 1942, tal y como afirmaba en la "Nota a la primera edición" que se incluyó en las solapas de la edición bonaerense de 1951: "Su acción discurre en Madrid -en 1942- y entre un torrente, o una colmena, de gentes que a veces son felices, y a veces, no"6.

De la censura religiosa de la novela se encargó Andrés de Lucas Casla, mientras que atendió a la censura laica Leopoldo Panero, buen amigo del novelista y prologuista de Pisando la dudosa luz del día. El censor eclesiástico tachó abundantemente la novela que, a su juicio, atacaba al dogma y a la moral y tenía un valor literario escaso. Cuando el censor da a conocer a Cela sus tachaduras, el novelista no las acepta y renuncia a la publicación, que, sin embargo, había intentado "salvar" Leopoldo Panero en atención al valor artístico y literario del texto (CACHERO, 1997, p. 102-103)7.

La colmena queda, pues, varada en febrero del 46. CJC va a desgajar del original de la novela -capítulo Vla materia narrativa del cuento "Unas gafas de color", que vio la luz en Arriba el 25 de abril de 1946 y posteriormente formó parte de El bonito crimen del carabinero y otras invenciones (José Janés, Barcelona, 1947). El tomo que contenía catorce narraciones breves -en su mayoría publicadas durante el 45- recogía dos relatos aparecidos en Arriba en 1946: "Las gafas de color" y "Dos cartas". En dicho tomo el relato desgajado de La colmena iba dedicado a Gonzalo Torrente Ballester.

Pero lo verdaderamente importante del tomo pasó desapercibido. $\mathrm{Y}$, sin embargo, es el contexto más explícito de la reconversión de unas viñetas del capítulo $\mathrm{V}$ de la novela inédita y prohibida en la narración "Las gafas de color". Se trata de un texto que abría el volumen y que CJC tituló "Notas para un prólogo". El texto presentaba dos características: las letras iniciales de los párrafos forman un acróstico y las reflexiones no tienen como tema las narraciones breves, sino lo que deberíamos llamar la naturaleza y el carácter de la novela según CJC. Vayamos por partes.

En el acróstico se puede leer: "Publico esto en pedazos porque tengo que comer. El cura que me censuró es un desdichado" (CJC, 1947, p. 9-15). Evidentemente los pedazos hacen referencia al "destrozo censorial" que había sufrido en manos del cura desdichado La colmena, porque, rigurosamente "pedazo" solo lo era el cuento dedicado a Torrente Ballester. Cela le explicó en 1955 al editor (entonces en tareas de entrevistador) Rafael Borràs Betriu que el acróstico era "su pequeña venganza contra el censor de turno" (BETRIU, 2003, p. 100). Por cierto, que cuando Borràs se lo comentó a Janés, que no había caído en la cuenta ocho años antes, al publicarse los relatos, éste sintió verdadera preocupación y "se quedó demudado"( BETRIU, 2003, p. 100). Anécdota que revela la escasísima trascendencia del acróstico, ya que "Notas para un prólogo" no volvió a ver la luz hasta la edición que de El bonito crimen del carabinero se hizo por las barcelonesas ediciones Picazo en $1972^{8}$. Se trata de un buen ejemplo del saber nadar y guardar la ropa que caracterizaba la conducta celiana de esos años tan procelosos como difíciles.

Las "Notas para un prólogo" son junto con "A vueltas con la novela", el artículo publicado en Ínsula (15-V-1947), las mejores reflexiones teóricas de CJC para entender la forja de la excepcional novela de 1951 a la par que sus ideas estéticas son válidas también para otras variantes narrativas, dado el descrédito con el que el joven maestro gallego trataba en esas latitudes a los géneros literarios (Cf. VÁZQUEZ, 1995, p. XXXV-XXXIX). Con muchísima sutileza Cela dice omitir "ciertas razones de algún alcance que, después de pensarlo mucho" (CJC, 1973, p. 543) le han decidido a contravenir su costumbre de no prologar sus libros ${ }^{9}$. Las razones que omite a buen seguro tienen que ver con la fragua de La colmena y su descalificación de novela poco trabada que había hecho el "cura desdichado" en su informe.

En las "Notas" se sostienen postulados fundamentales de su arte narrativo. La novela es un río que recoge el ritmo vital, la cadencia de las vidas humanas:

Tiembla en la novela la sustancia misma de la vida, como tiembla en el río la esencia misma del movimiento. En tanto que camino la vida, cabe pensar que las páginas que caminen a su ritmo son las páginas de una novela (CJC, 1973, p. 544).

El oficio sin metro del novelista es captar lo radicalmente vital con un écran que rechaza las lunas planas, es aprehender el ruido y la furia, el torrente o la colmena de la vida, es hacer temblar en el relato la sombra misma del hombre:

\footnotetext{
6 CJC, "Nota a la primera edición". Cito por CJC, La colmena, p. 145.

7 Los dictámenes de los censores pueden verse en José María Martínez Cachero, La novela española entre 1936 y el fin de siglo. Historia de una aventura.

8 Se reproduce "Notas para un prólogo" pero sin conformar el acróstico. Son simplemente trece notas. Para más información debe verse el utilísimo tomito de Fernando Huarte Morton, Los cuentos de "El bonito crimen del carabinero" de CJC.

9 Es una verdad a medias. Cierto que las primeras ediciones de $L a$ familia de Pascual Duarte (1942), Pabellón de reposo (1943) y Nuevas andanzas y desventuras de Lazarillo de Tormes (1944) no llevan prólogo, pero la bella edición del Pascual de Ediciones del Zodíaco (Barcelona, 1946) - que es la cuarta- contiene una censurada "Breve historia de esta novela" (p. IX-XXV), de gran importancia e interés.
} 
Ir a remolque, hablar como se habla, respirar como respiran los que están vivos y acaban por dejar de respirar. Coger la vida y estrujarla contra nuestro corazón. He ahí la labor del novelista (CJC, 1973, p. 547).

Cela, provisto de una carta de marear que atendía a la gran tradición del 98 y a Ortega, navegó hacia una novela que representaba, desde la mirada y la memoria, la morada vital de la sociedad española:

Una novela es, pensémoslo bien, la fe de vida de un pueblo y de un momento, interpretados ambos literariamente. El personaje es el fedatario, nunca el documento mismo (CJC, 1973, p. 544).

El novelista es el notario de la conciencia de un pueblo y de un momento. La novela es el pulso de esa fluencia vital, de ese pedazo de vida. El personaje es el fedatario. Se trata de un haz de reflexiones que laten en un texto contemporáneo de la publicación de La colmena:

El escritor es el notario de la conciencia de su tiempo $\mathrm{y}$ de su mundo, y a la conciencia hay que tomarle el pulso donde está, a ras de tierra, pegada a la corteza de la tierra, esa caja de resonancia donde se escucha, isócrono y amargo, el cruento retumbar de los corazones (CJC, 1989, p. 570-571).

CJC defendía en esas notas prologales su quehacer como el sumatorio de la mirada y la memoria volcadas sobre cualquier paisaje vital. O dicho de otro modo, estrujar contra su corazón a los seres anónimos del torrente de la vida madrileña de 1943 para oírles respirar.

\section{III}

Para finales de la primavera del 45 el proyecto de $L a$ colmena va a tener su primera presentación pública. Se produce en Madrid. Aprovechando la inauguración en el Museo Nacional de Arte Moderno de una exposición de estampas del pintor madrileño Juan Esplandíu, el 19 de junio, Cela lee los dos primeros capítulos el 28 de junio y el 3 de agosto publica en Arriba un brillante artículo, "El alma de Madrid en 34 acuarelas", clave para entender el tejido novelesco que anda en el telar del joven escritor.

El episodio, la primera lectura pública de un texto que conforma el momento inicial de la futura obra maestra, ofrece datos decisivos. De un lado -y gracias a la correspondencia con el editor barcelonés Maristanysabemos que Cela anda tejiendo una novela larga (le habla de cerca de mil páginas, que serían a buen seguro el conjunto del ciclo Caminos inciertos); que ese trabajo lo combina con la preparación de las dos novelas que apalabró (y en parte cobró) con Saturnino Calleja; que está preocupado por la dureza de la censura, dado que la novela que presentó en el Museo Nacional de Arte Moderno no es una novela rosa; que la presentación tuvo un notable éxito (según Informaciones del 29 de junio al acto "asistieron numerosísimos artistas y escritores"); y finalmente, que la futura obra maestra -que editará las Ediciones del Zodiaco de Maristany- se llama ahora -junio de 1945- La ciudad llagada y que forma parte del ciclo Vagando por los caminos inciertos. ${ }^{10}$

De otra parte, el artículo celiano sobre Juan Esplandíu revela su abierta admiración por las acuarelas que "dan al visitante la cierta novela de Madrid, la tremenda, abigarrada, cómica novela de nuestra capital" (CJC, 1976, p. 350). Y a la par, el deseo implícito de convertirse en el complemento de Esplandíu, el pintor que años después, 1966, ilustrará los textos de su libro Madrid. La simbiosis entre Cela y Esplandíu la ha mostrado con delicadeza la profesora Marta Cristina en su artículo "Tres ventanas sobre Madrid" (Cf. CARBONELL, 2011). Aquí y ahora sólo me cabe invocar el artículo de Cela de 1945: "El Madrid de nuestros días, que busca el novelista que escriba su novela, ha encontrado el pintor que lo supo retratar." (CJC, 1976, p. 251)

Cela tenía una excelente información sobre los quehaceres de Esplandíu, en la línea de Gutiérrez Solana, a quien dedicará su discurso de ingreso en la Real Academia Española en 1957, La obra literaria del pintor Solana. Ahora, en 1945, podría desdoblarse en la obra pictórica de Esplandíu y la obra narrativa que estaba naciendo. Madrid había encontrado su novelista, se estaba gestando.

La segunda lectura pública en Madrid de la excepcional novela de 1951 tuvo lugar durante el invierno del 46. Una nota de $A B C$ del 18 de enero del 46 reza así: "Se inauguró la Exposición de Pinturas Porcar con 47 obras y el escritor Camilo José Cela leyó en la Exposición Facetas del Arte Moderno Español, dos capítulos de su nueva novela La colmena". Como se advierte la novela ya gozaba de su título definitivo, dato confirmado por una carta a Saturnino Calleja, director de Ediciones "La Nave", donde Cela había publicado Nuevas andanzas y desventuras de Lazarillo de Tormes (1944), fechada el 14 de enero del 46:

He acabado una novela que titulo La colmena y que, dadas sus características, no encaja en 'La Nave'. Con lo que saque de ella pienso pagarle a usted. Ni que decir tiene que mis dos libros anunciados en ' $\mathrm{La}$ Nave' -Las aguas tranquilas y Un marino mercanteserán suyas, si a usted le parecen bien, tan pronto como las termine. ${ }^{11}$

\footnotetext{
${ }^{10}$ Ver la carta de CJC a Maristany en Adolfo Sotelo Vázquez, "La colmena, primeros pasos", Cuadernos Hispanoamericanos, 761 (2013), p.130-131.

11 Saturnino Calleja le había anticipado 7500 pesetas, que CJC devolvió religiosamente. Cabe indicar que las dos novelas que le promete nunca se escribieron, aunque constaban en las "Obras del mismo autor" de la primera edición de Nuevas andanzas y desventuras de Lazarillo de Tormes.
} 
El lugar donde CJC leyó la novela fue la Galería Buchholz -librería y sala de exposiciones-dirigida por Enrique Azcoaga, amigo entrañable de Camilo José en esos años. El día de la lectura fue el jueves 17 de enero, jornada en la que nevó abundantemente en Madrid.

El 23 de octubre de 1945 Camilo José Cela llega a Barcelona procedente de Madrid, donde reside, tras su matrimonio con Charo Conde, en un piso de la calle de Alcalá, muy cercano a la plaza de toros de las Ventas. Su taller de creación trabaja a toda máquina y durante ese mismo año habían visto la luz Pisando la dudosa luz del día. Poemas de una adolescencia cruel (que edita con primor Carlos F. Maristany en las barcelonesas Ediciones del Zodíaco), Esas nubes que pasan (volumen de relatos que publicó Afrodisio Aguado en Madrid), y Mesa revuelta (colección de artículos ya publicados, que ve la luz en las prensas madrileñas de Sagitario). Completaban estas publicaciones sus tres novelas primeras: La familia de Pascual Duarte (1942), Pabellón de reposo (1943) y Nuevas andanzas y desventuras del Lazarillo de Tormes (1944).

CJC tiene entre manos una infinidad de proyectos, tal y como anunciaba la sobrecubierta de la edición de El nuevo Lazarillo, entre ellos dos novelas que nunca fueron: El marino mercante ${ }^{12}$ y Las aguas tranquilas. Sin embargo, Cela mantiene notable sigilo sobre la obra que tiene en marcha y que se llama Caminos inciertos, y cuyo primer libro es La colmena. Precisamente el motivo principal del viaje a Barcelona es -como recordaban las notas informativas de diversos periódicos barcelonesesinaugurar el martes 30 de octubre el curso de conferencias que organizaba la Delegación de Educación Popular en el Ateneo Barcelonés. La Vanguardia (28-X) añadía que el tema de la sesión inaugural sería la "lectura de unos capítulos de su novela inédita Caminos inciertos. El anuncio de esta lectura ha despertado vivo interés en los círculos literarios y artísticos barceloneses"13. No podía ser de otro modo, CJC, invitado por el falangista Luys Santa Marina, presidente del Ateneo y consejero nacional del Movimiento, iba a leer por primera vez en

\footnotetext{
12 En una breve entrevista que concedió a Fotos (23-X-1944) para una sección titulada "Los escritores y el mar", Camilo José reconoce que está escribiendo una novela marinera: "En la actualidad trabajo en una novela -Un marino mercante-, cuya acción transcurre, casi constantemente, en el mar o en su más próxima orilla. En ella relato las andanzas de mi tío don Evaristo Montenegro de Cela, elegante prosista y capitán mercante retirado, gran tipo familiar que murió precisamente el año que yo nací." En La Rosa, libro primero de La Cucaña. Memorias de Camilo José Cela (Barcelona, Destino, 1959) el escritor no menciona a este pariente de la familia de su padre, personaje central de una ficción nonata.

$13 \mathrm{Cf}$. "El señor Cela ha sido invitado a inaugurar el ciclo de conferencias del Ateneo Barcelonés, acto en el que nos dará a conocer las primicias de su última novela" (La Prensa, 26-X-1945). El 30 de octubre, Diario de Barcelona, El Correo Catalán Solidaridad Nacional anuncian con el mismo texto la conferencia de CJC: "El ilustre novelista leerá unos capítulos de su novela inédita Caminos inciertos".
}

público unos capítulos de la novela, que tras una larga y ardua pelea con la censura, vería la luz en Buenos Aires (1951), convirtiéndose en una de las obras maestras de la narrativa española del siglo XX.

$\mathrm{Al}$ margen del programa de visitas, conversaciones, firma de ejemplares, etc., que le había preparado Juan Ramón Masoliver, la lectura de dos capítulos de $L a$ colmena es, sin duda, el hecho más relevante de la estancia de Cela en Barcelona. Solidaridad Nacional (31-X) informaba de quienes presidieron el acto: Santa Marina, José Pardo (del Departamento de Propaganda), Luis Monreal (comisario del Patrimonio Artístico), José María Junyent (por el Ayuntamiento), Rodríguez Codolá (por las Reales Academias de Ciencias y Artes y de Bellas Artes de San Jorge), Martín de Riquer (por la Real Academia de Buenas Letras), Castro y Calvo (decano de la Facultad de Filosofía y Letras) y los miembros de la junta del Ateneo: Masoliver, Vela, Juliá y Caralt. De una presidencia tan nutrida y de una magnífica fotografía de Pérez de Rozas que acompañaba la información, se deduce el escenario político-social que albergó la primera lectura de La colmena. La nota informativa de La Vanguardia (31-X) recordaba que antes de la lectura de los capítulos I y II de la novela inédita, "Cela trazó un breve preámbulo en el que expuso su intención estética al confeccionar la novela, que ha sido escrita -dijocon técnica de contrapunto. La obra se desarrolla en el Madrid actual, con acciones diversas y ágil movimiento de personajes". La información de Solidaridad Nacional (31-X) se extendía pormenorizando el argumento de los capítulos leídos de modo fragmentario: "El primer capítulo leído presenta el ambiente de un café popular de la calle de Fuencarral [...] En el segundo capítulo, usa Cela una técnica dinámica y original, en virtud de la cual presenta entremezcladas acciones diversas y simultáneas a través de las cuales se empieza a atar los cabos con los que se ha de tramar el argumento de la novela".

El Correo Catalán, El Noticiero Universal y La Prensa recogieron también las incidencias del acto del Ateneo. El semanario Destino -al que Cela estaría muy vinculado en los años venideros- consignaba en la sección "Entre líneas" (3-XI), que redactó Masoliver: "Se sabe que Caminos inciertos será editado en su día -un día del año próximo- por Fernando Maristany, unos de los hombres que más alto han puesto el buen nombre de los editores barceloneses". En efecto, Carlos F. Maristany, de ediciones del Zodíaco (quien publicaría en el 46 la cuarta edición del Pascual Duarte, la primera barcelonesa, con prólogo de Gregorio Marañón) presentó, junto con el escritor, La colmena a la censura el 7 de enero de 1946. La censura la prohibió y el propio escritor le instó a presentar de nuevo el texto, primera parte del libro primero Caminos inciertos, de La Tarea infinita (novela), 
como libro de bibliófilo para poder salvar los escollos de la censura. Cela recordó en 1965 ("Historia incompleta de unas páginas zarandeadas") lo sucedido: "El 27 de febrero solicitó el editor el oportuno permiso para una tirada de características especiales, de lujo y reducida; fue también denegada, en oficio del 9 de marzo". La publicación de la novela que había dado a conocer el 30 de octubre del 45 en la histórica sesión del Ateneo Barcelonés quedará varada al finalizar el invierno del 46. Su azarosa prehistoria habría de concluir en Buenos Aires en 1951.

Durante la estancia Cela asistió a comidas con editores y escritores, en las que trató de abrir camino a sus proyectos, visitó Sant Cugat y Sitges, conoció personalmente a González Ruano y creó un pequeño círculo de amigos barceloneses, que quizás quede pertinentemente enumerado por los comensales que asistieron, después de la sesión del Ateneo, a la cena en el restaurante "Las siete puertas"14: Santa Marina, Caralt, Vela, Masoliver, Arbó, Monreal, Serrano, Utrillo, Garcés, Segalá, Agustí, Zúñiga, Riquer y Martínez Barbeitio. Círculo barcelonés al que habría que sumar el confinado (en Llavaneras) Ridruejo, los editores Maristany y Janés, y González Ruano y Álvaro Ruibal, que no asistieron a la cena.

Buen colofón del tránsito barcelonés fue una notable semblanza del escritor a cargo de Ángel Zúñiga en Liceo (XI, 1945), un excelente artículo de Juan Ramón Masoliver en La Vanguardia (1-XI) ${ }^{15}$ y una entrevista de Manuel del Arco en El Correo Catalán (30-X), en la que a la pregunta de qué es la novela, CJC contestaba: "Acepto la definición de Stendhal: es un espejo que pasea a lo largo del camino".

\section{Referencias}

BETRIU, Rafael Borràs. La batalla de Waterloo. Memorias de un editor (I). Barcelona: Ediciones B, 2003.

CACHERO, José María Martínez. La novela española entre 1936 y el fin de siglo. Historia de una aventura. Madrid: Castalia, 1997.

CARBONELL. Marta Cristina. Tres ventanas sobre Madrid. Anuario de Estudios celianos. 2011. p. 53-67.

CJC. Algo sobre el género y los géneros. La Voz del Sur (Cádiz, 4-VI-1949); Obra completa. Barcelona: Destino, 1928. t. XII.

CJC. Algunas notas en torno al concepto de novela. Obra completa. Barcelona: Destino, 1978.
CJC. El alma de Madrid en 34 acuarelas. Glosa del mundo en torno. Artículos, 1. Obra completa. Barcelona: Destino, 1976. t. IX.

CJC. Elogio del mirón. La Vanguardia (15-X-1952); Obra completa. Barcelona: Destino, 1978. t.X.

CJC. Elogio del mirón, La Vanguardia (15-X-1952); Obra completa. Barcelona: Destino, 1978. t. X.

CJC. Esa ventana abierta sobre cualquier paisaje. Arriba (5-IX1950); Obra completa. Barcelona: Destino, 1978. t. X.

CJC. Esa ventana abierta sobre cualquier paisaje. Arriba (5-IX1950); Obra completa. Barcelona: Destino, 1978. t. X.

CJC. La galera de la literatura (Ínsula, marzo 1951). Glosa del mundo en torno. Obra completa. Barcelona: Destino, 1989. t. XII.

CJC. La noche, ese misterio. La Vanguardia (15-VI-1950); Obra completa. Barcelona: Destino, 1978. t. X.

CJC. Notas para un prólogo. Obra completa. Barcelona: Destino, 1973. t. I.

CJC. Sobre España, los españoles y lo español (1959). Obra completa. Barcelona: Destino, 1978. t. XII.

CJC. Sobre las artes de novelar. Correo Literario (1-V-1952); Obra completa. Barcelona: Destino, 1978. t. XII.

CJC. El bonito crimen del carabinero y otras invenciones. Barcelona: José Janés, 1947.

CJC. Viaje a la Alcarria; Obra completa. Barcelona: Destino, 1965. t. IV. (Viajes por España, I).

MORTON, Fernando Huarte. Bibliografía celiana: el manuscrito de La colmena. La obra literaria de Camilo José Cela (IV curso de verano). Iria Flávia: Fundación Camilo José Cela, 2000.

MORTON, Fernando Huarte. Los cuentos de "El bonito crimen del carabinero" de CJC. O Tabeiron Namorado. Iria Flavia: Fundación Camilo José Cela, 1998. t. VI.

UNAMUNO, Miguel de. Tres novelas ejemplares y un prólogo (ed. Ciríaco Morón Arroyo). Madrid: Espasa Calpe (Austral), 1990.

VÁZQUEZ, Adolfo Sotelo. "Introducción" a CJC. La familia de Pascual Duarte. Barcelona: Destino, 1995.

VÁZQUEZ, Adolfo Sotelo. La colmena, primeros pasos. Cuadernos Hispanoamericanos, 761, 2013.

Recebido: 20/12/2015

Aprovado: 13/01/2016

Contato: sotelo@ub.edu

\footnotetext{
14 Tomo la información de la habitual columna "Tertulia en la Rambla", que firmaba "Laercio", seudónimo del escritor y crítico de arte Luis Monreal Tejada, en Solidaridad Nacional (2-IX-1945).

15 Ambos textos se pueden leer en mi libro, Camilo José Cela. Perfiles de un escritor, Sevilla, Renacimiento, 2008, p. 75-78.
} 